\title{
Sharp $H_{p}-L_{p}$ type inequalities of weighted maximal operators of Vilenkin-Nörlund means and its applications
}

\author{
Lasha Baramidze', Lars-Erik Persson ${ }^{2,3^{*}}$, George Tephnadze ${ }^{1,2}$ and Peter Wall ${ }^{2}$
}

"Correspondence: larserik@ltu.se ${ }^{2}$ Department of Engineering Sciences and Mathematics, Luleå University of Technology, Luleå, 971 87, Sweden

${ }^{3} \mathrm{UiT}$, The Artic University of Norway, P.O. Box 385, Narvik, 8505, Norway Full list of author information is available at the end of the article

\begin{abstract}
We prove and discuss some new $H_{p}-L_{p}$ type inequalities of weighted maximal operators of Vilenkin-Nörlund means with monotone coefficients. It is also proved that these inequalities are the best possible in a special sense. We also apply these results to prove strong summability for such Vilenkin-Nörlund means. As applications, both some well-known and new results are pointed out.
\end{abstract}

MSC: $42 \mathrm{C} 10 ; 42 \mathrm{~B} 25$

Keywords: inequalities; Vilenkin system; Vilenkin group; Nörlund means; martingale Hardy space; $L_{p}$ spaces; maximal operator; Vilenkin-Fourier series

\section{Introduction}

The definitions and notations used in this introduction can be found in our next section. In the one-dimensional case the weak $(1,1)$-type inequality for maximal operator of Fejér means $\sigma^{*} f:=\sup _{n \in \mathbb{N}}\left|\sigma_{n} f\right|$ can be found in Schipp [1] for Walsh series and in Pál, Simon [2] for bounded Vilenkin series. Fujji [3] and Simon [4] verified that $\sigma^{*}$ is bounded from $H_{1}$ to $L_{1}$. Weisz [5] generalized this result and proved boundedness of $\sigma^{*}$ from the martingale space $H_{p}$ to the Lebesgue space $L_{p}$ for $p>1 / 2$. Simon [6] gave a counterexample, which shows that boundedness does not hold for $0<p<1 / 2$. In the case $p=1 / 2$ a counterexample with respect to Walsh system was given by Goginava [7] and for the bounded Vilenkin system was proved by Tephnadze [8]. Weisz [9] proved that the maximal operator of the Fejér means $\sigma^{*}$ is bounded from the Hardy space $H_{1 / 2}$ to the space weak- $L_{1 / 2}$.

Weisz [10] proved that the maximal operator of Cesàro means $\sigma^{\alpha, *} f:=\sup _{n \in \mathbb{N}}\left|\sigma_{n}^{\alpha} f\right|$ is bounded from the martingale space $H_{p}$ to the space $L_{p}$ for $p>1 /(1+\alpha)$. Goginava [11] gave a counterexample, which shows that boundedness does not hold for $0<p \leq 1 /(1+\alpha)$. Simon and Weisz [12] showed that the maximal operator $\sigma^{\alpha, *}(0<\alpha<1)$ of the $(C, \alpha)$ means is bounded from the Hardy space $H_{1 /(1+\alpha)}$ to the space weak- $L_{1 /(1+\alpha)}$. In [13] and [14] it was also proved that the maximal operator

$$
\widetilde{\sigma}_{p}^{\alpha, *}:=\sup _{n \in \mathbb{N}}\left|\sigma_{n}^{\alpha} f\right| /\left((n+1)^{1 / p-\alpha-1} \log ^{(1+\alpha)[p+\alpha(1+\alpha)]}(n+1)\right)
$$

(c) 2016 Baramidze et al. This article is distributed under the terms of the Creative Commons Attribution 4.0 International License (http://creativecommons.org/licenses/by/4.0/), which permits unrestricted use, distribution, and reproduction in any medium, provided you give appropriate credit to the original author(s) and the source, provide a link to the Creative Commons license, and indicate if changes were made. 
is bounded from the Hardy space $H_{p}$ to the Lebesgue space $L_{p}$, where $0<p \leq 1 /(1+\alpha)$. Moreover, the rate of the weights $\left\{(n+1)^{1 / p-\alpha-1} \log ^{(1+\alpha)[p+\alpha(1+\alpha)]}(n+1)\right\}_{n=1}^{\infty}$ in $n$th Cesàro mean is given exactly.

It is well known that Vilenkin systems do not form bases in the space $L_{1}\left(G_{m}\right)$. Moreover, there is a function in the Hardy space $H_{1}\left(G_{m}\right)$, such that the partial sums of $f$ are not bounded in $L_{1}$-norm. Simon [15] (for unbounded Vilenkin systems in the case when $p=1$ see [16] and for $0<p<1$ another proof was pointed out in [17]) proved that there exists an absolute constant $c_{p}$, depending only on $p$, such that

$$
\frac{1}{\log ^{[p]} n} \sum_{k=1}^{n} \frac{\left\|S_{k} f\right\|_{p}^{p}}{k^{2-p}} \leq c_{p}\|f\|_{H_{p}}^{p} \quad(0<p \leq 1)
$$

for all $f \in H_{p}$ and $n \in \mathbb{N}_{+}$, where $[p]$ denotes the integer part of $p$. In [18] for Walsh system and in [19] with respect to bounded Vilenkin system it was proved that sequence $\left\{1 / k^{2-p}\right\}_{k=1}^{\infty}(0<p<1)$ in (1) cannot be improved.

In [20] it was proved that there exists an absolute constant $c_{p}$, depending only on $p$, such that

$$
\frac{1}{\log ^{[1 / 2+p]} n} \sum_{k=1}^{n} \frac{\left\|\sigma_{k} f\right\|_{p}^{p}}{k^{2-2 p}} \leq c_{p}\|f\|_{H_{p}}^{p} \quad(0<p \leq 1 / 2, n=2,3, \ldots) .
$$

An analogous result for $(C, \alpha)(0<\alpha<1)$ means when $p=1 /(1+\alpha)$ was generalized in [13] and when $0<p<1 /(1+\alpha)$ it was proved in [14]. In particular, the following inequality:

$$
\frac{1}{\log ^{[\alpha /(1+\alpha)+p]} n} \sum_{k=1}^{n} \frac{\left\|\sigma_{k}^{\alpha} f\right\|_{p}^{p}}{k^{2-(1+\alpha) p}} \leq c_{p}\|f\|_{H_{p}}^{p} \quad(0<p \leq 1 /(1+\alpha), n=2,3, \ldots)
$$

holds.

Móricz and Siddiqi [21] investigated the approximation properties of some special Nörlund mean of the $L_{p}$ function in norm. For more information on Nörlund means, see the paper of Blahota and Gát [22] and Nagy [23] (see also [24, 25], and [26]).

In [27] for $p=1 /(1+\alpha)$ and in [28] for $0<p<1 /(1+\alpha)$ there was proved that for every $f \in H_{p}$ and for every Nörlund mean $t_{n} f$, generated by the non-increasing sequence $\left\{q_{n}\right.$ : $n \geq 0\}$, satisfying the conditions

$$
n^{\alpha} / Q_{n}=O(1), \quad \text { as } n \rightarrow \infty,
$$

and

$$
\left(q_{n}-q_{n+1}\right) / n^{\alpha-2}=O(1), \quad \text { as } n \rightarrow \infty,
$$

there exists an absolute constant $c_{\alpha, p}$ such that

$$
\frac{1}{\log ^{[\alpha /(1+\alpha)+p]} n} \sum_{k=1}^{n} \frac{\left\|t_{k} f\right\|_{p}^{p}}{k^{2-(1+\alpha) p}} \leq c_{\alpha, p}\|f\|_{H_{p}}^{p} \quad(n=2,3, \ldots)
$$


and

$$
\left\|\sup _{n \in \mathbb{N}}\left|t_{n} f\right| /\left((n+1)^{1 / p-\alpha-1} \log ^{(1+\alpha)[p+\alpha(1+\alpha)]}(n+1)\right)\right\|_{H_{p}} \leq c_{\alpha, p}\|f\|_{H_{p}}
$$

In [29] it was proved that in the endpoint case $p=1 /(1+\alpha)$ both (3) and (4) conditions are sharp in a special sense.

In this paper we investigate the case when $0<p<1 /(1+\alpha)$ and prove inequalities (5) and (6) for $f \in H_{p}$ and Vilenkin-Nörlund means with non-increasing coefficients, but with weaker conditions than (3) and (4), which give possibility to prove analogous results for the wider class of Vilenkin-Nörlund means when $0<p<1 /(1+\alpha)$. As applications, both some well-known and new results are pointed out.

This paper is organized as follows: In order not to disturb our discussions later on some definitions and notations are presented in Section 2. The main results can be found in Section 3. For the proofs of the main results we need some lemmas, both well known, but also some new ones of independent interest. These results are presented in Section 4 . The detailed proofs are given in Section 5. Some well-known and new consequences of our main results are presented in Section 6.

\section{Definitions and notations}

Denote by $\mathbb{N}_{+}$the set of the positive integers, $\mathbb{N}:=\mathbb{N}_{+} \cup\{0\}$. Let $m:=\left(m_{0}, m_{1}, \ldots\right)$ be a sequence of the positive integers not less than 2 . Denote by

$$
Z_{m_{k}}:=\left\{0,1, \ldots, m_{k}-1\right\}
$$

the additive group of integers modulo $m_{k}$.

Define the group $G_{m}$ as the complete direct product of the groups $Z_{m_{i}}$ with the product of the discrete topologies of the $Z_{m_{j}}$.

The direct product $\mu$ of the measures

$$
\mu_{k}(\{j\}):=1 / m_{k} \quad\left(j \in Z_{m_{k}}\right)
$$

is the Haar measure on $G_{m}$ with $\mu\left(G_{m}\right)=1$.

In this paper we discuss bounded Vilenkin groups, i.e. the case when $\sup _{n} m_{n}<\infty$.

The elements of $G_{m}$ are represented by the sequences

$$
x:=\left(x_{0}, x_{1}, \ldots, x_{j}, \ldots\right) \quad\left(x_{j} \in Z_{m_{j}}\right)
$$

It is easy to give a base for the neighborhood of $G_{m}$ :

$$
I_{0}(x):=G_{m}, \quad I_{n}(x):=\left\{y \in G_{m} \mid y_{0}=x_{0}, \ldots, y_{n-1}=x_{n-1}\right\},
$$

where $x \in G_{m}, n \in \mathbb{N}$.

Denote $I_{n}:=I_{n}(0)$ for $n \in \mathbb{N}_{+}$, and $\overline{I_{n}}:=G_{m} \backslash I_{n}$.

$$
\overline{I_{N}}=\left(\bigcup_{k=0}^{N-2 m_{k}-1} \bigcup_{s_{k}=1}^{N-1} \bigcup_{l=k+1}^{m_{l}-1} \bigcup_{s_{l}=1} I_{l+1}\left(s_{k} e_{k}+s_{l} e_{l}\right)\right) \cup\left(\bigcup_{k=1}^{N-1 m_{k}-1} \bigcup_{s_{k}=1}^{-1} I_{N}\left(s_{k} e_{k}\right)\right) .
$$


If we define the so-called generalized number system based on $m$ in the following way:

$$
M_{0}:=1, \quad M_{k+1}:=m_{k} M_{k} \quad(k \in \mathbb{N})
$$

then every $n \in \mathbb{N}$ can be uniquely expressed as $n=\sum_{j=0}^{\infty} n_{j} M_{j}$, where $n_{j} \in Z_{m_{j}}\left(j \in \mathbb{N}_{+}\right)$and only a finite number of the $n_{j}$ differ from zero.

Next, we introduce on $G_{m}$ an orthonormal system which is called the Vilenkin system. At first, we define the complex-valued function $r_{k}(x): G_{m} \rightarrow \mathbb{C}$, the generalized Rademacher functions, by

$$
r_{k}(x):=\exp \left(2 \pi i x_{k} / m_{k}\right) \quad\left(i^{2}=-1, x \in G_{m}, k \in \mathbb{N}\right) .
$$

Now, define the Vilenkin system $\psi:=\left(\psi_{n}: n \in \mathbb{N}\right)$ on $G_{m}$ as

$$
\psi_{n}(x):=\prod_{k=0}^{\infty} r_{k}^{n_{k}}(x) \quad(n \in \mathbb{N}) .
$$

Specifically, we call this system a Walsh-Paley system when $m \equiv 2$.

The norms (or quasi-norms) of the spaces $L_{p}\left(G_{m}\right)$ and weak- $L_{p}\left(G_{m}\right)(0<p<\infty)$ are, respectively, defined by

$$
\|f\|_{p}^{p}:=\int_{G_{m}}|f|^{p} d \mu, \quad\|f\|_{\text {weak }-L_{p}}^{p}:=\sup _{\lambda>0} \lambda^{p} \mu(f>\lambda) .
$$

The Vilenkin system is orthonormal and complete in $L_{2}\left(G_{m}\right)$ (see [30]).

Next, we introduce analogs of the usual definitions in Fourier-analysis. If $f \in L_{1}\left(G_{m}\right)$ we can define the Fourier coefficients, the partial sums of the Fourier series, the Dirichlet kernels with respect to the Vilenkin system in the usual manner:

$$
\begin{aligned}
& \widehat{f}(n):=\int_{G_{m}} f \bar{\psi}_{n} d \mu(n \in \mathbb{N}), \\
& S_{n} f:=\sum_{k=0}^{n-1} \widehat{f}(k) \psi_{k}, \quad D_{n}:=\sum_{k=0}^{n-1} \psi_{k} \quad\left(n \in \mathbb{N}_{+}\right),
\end{aligned}
$$

respectively.

Recall that

$$
D_{M_{n}}(x)= \begin{cases}M_{n}, & \text { if } x \in I_{n}, \\ 0, & \text { if } x \notin I_{n},\end{cases}
$$

and

$$
D_{n}=\psi_{n}\left(\sum_{j=0}^{\infty} D_{M_{j}} \sum_{k=m_{j}-n_{j}}^{m_{j}-1} r_{j}^{k}\right),
$$

for $n=\sum_{i=0}^{\infty} n_{i} M_{i}$.

The $\sigma$-algebra generated by the intervals $\left\{I_{n}(x): x \in G_{m}\right\}$ will be denoted by $\digamma_{n}(n \in \mathbb{N})$. Denote by $f=\left(f^{(n)}, n \in \mathbb{N}\right)$ a martingale with respect to $\digamma_{n}(n \in \mathbb{N})$ (for details see e.g. [31]). 
The maximal function of a martingale $f$ is defined by

$$
f^{*}=\sup _{n \in \mathbb{N}}\left|f^{(n)}\right|
$$

For $0<p<\infty$ the Hardy martingale spaces $H_{p}\left(G_{m}\right)$ consist of all martingales for which

$$
\|f\|_{H_{p}}:=\left\|f^{*}\right\|_{p}<\infty
$$

If $f=\left(f^{(n)}, n \in \mathbb{N}\right)$ is a martingale, then the Vilenkin-Fourier coefficients must be defined in a slightly different manner:

$$
\widehat{f}(i):=\lim _{k \rightarrow \infty} \int_{G_{m}} f^{(k)} \bar{\psi}_{i} d \mu
$$

Let $\left\{q_{k}: k \geq 0\right\}$ be a sequence of nonnegative numbers. The $n$th Nörlund means for a Fourier series of $f$ is defined by

$$
t_{n} f:=\frac{1}{Q_{n}} \sum_{k=1}^{n} q_{n-k} S_{k} f,
$$

where

$$
Q_{n}:=\sum_{k=0}^{n-1} q_{k} .
$$

A representation

$$
t_{n} f(x)=\int_{G_{m}} f(t) F_{n}(x-t) d \mu(t)
$$

plays a central role in the sequel, where

$$
F_{n}:=\frac{1}{Q_{n}} \sum_{k=1}^{n} q_{n-k} D_{k}
$$

is the so-called Nörlund kernel.

We say that the Nörlund mean $t_{n}$ is of $(N, \alpha)$ type if

$$
\frac{n^{\alpha}}{Q_{n}}=O(1), \quad \text { as } n \rightarrow \infty
$$

and for any $\varepsilon>0$, we have

$$
\frac{Q_{n}}{n^{\alpha+\varepsilon}} \rightarrow 0, \quad \text { as } n \rightarrow \infty
$$

For our further investigation it is much more convenient to replace condition (12) by its equivalent one:

$$
Q_{n} \leq c_{\alpha} n^{\alpha} \varphi_{n}, \quad \text { where } \varlimsup_{j \rightarrow \infty} \frac{\varphi_{j}}{j^{\varepsilon}}=0 \text {, for every } \varepsilon>0 .
$$


We always assume that $q_{0}>0$ and $\lim _{n \rightarrow \infty} Q_{n}=\infty$. In this case it is well known that the summability method generated by $\left\{q_{k}: k \geq 0\right\}$ is regular if and only if

$$
\lim _{n \rightarrow \infty} \frac{q_{n-1}}{Q_{n}}=0
$$

Concerning this fact and related basic results, we refer to [32].

If $q_{n} \equiv 1$, then we get the $n$th Fejér mean and the Fejér kernel

$$
\sigma_{n} f:=\frac{1}{n} \sum_{k=1}^{n} S_{k} f, \quad K_{n}:=\frac{1}{n} \sum_{k=1}^{n} D_{k},
$$

respectively.

Let $t, n \in \mathbb{N}$. It is well known that (see [33])

$$
K_{M_{n}}(x)= \begin{cases}0, & \text { if } x-x_{t} e_{t} \notin I_{n}, x \in I_{t} \backslash I_{t+1}, \\ \frac{M_{t}}{1-r_{t}(x)}, & \text { if } x-x_{t} e_{t} \in I_{n}, x \in I_{t} \backslash I_{t+1}, \\ \left(M_{n}+1\right) / 2, & \text { if } x \in I_{n} .\end{cases}
$$

The $(C, \alpha)$-means of the Vilenkin-Fourier series are defined by

$$
\sigma_{n}^{\alpha} f:=\frac{1}{A_{n}^{\alpha}} \sum_{k=1}^{n} A_{n-k}^{\alpha-1} S_{k} f
$$

where

$$
A_{0}^{\alpha}:=0, \quad A_{n}^{\alpha}:=\frac{(\alpha+1) \cdots(\alpha+n)}{n !}, \quad \alpha \neq-1,-2, \ldots
$$

For the martingale $f$ we consider the following maximal operators:

$$
\sigma^{*} f:=\sup _{n \in \mathbb{N}}\left|\sigma_{n} f\right|, \quad \sigma^{\alpha, *} f:=\sup _{n \in \mathbb{N}}\left|\sigma_{n}^{\alpha} f\right|, \quad t^{*} f:=\sup _{n \in \mathbb{N}}\left|t_{n} f\right| .
$$

We also consider the following weighted maximal operators:

$$
\begin{aligned}
& \widetilde{\sigma}_{p}^{*}:=\sup _{n \in \mathbb{N}}\left|\sigma_{n} f\right| /\left((n+1)^{1 / p-2} \log ^{2[p+1 / 2]}(n+1)\right) \quad(0<p \leq 1 / 2), \\
& \widetilde{\sigma}_{p}^{\alpha, *}:=\sup _{n \in \mathbb{N}}\left|\sigma_{n}^{\alpha} f\right| /\left((n+1)^{1 / p-\alpha-1} \log ^{(1+\alpha)[p+\alpha(1+\alpha)]}(n+1)\right) \quad(0<p \leq 1 /(1+\alpha)), \\
& \widetilde{t}_{p}^{*}:=\sup _{n \in \mathbb{N}}\left|t_{n} f\right| /\left((n+1)^{1 / p-\alpha-1} \log ^{(1+\alpha)[p+\alpha(1+\alpha)]}(n+1)\right) \quad(0<p \leq 1 /(1+\alpha)) .
\end{aligned}
$$

A bounded measurable function $a$ is a p-atom, if there exists an interval $I$, such that

$$
\int_{I} a d \mu=0, \quad\|a\|_{\infty} \leq \mu(I)^{-1 / p}, \quad \operatorname{supp}(a) \subset I .
$$

\section{The main results}

Our sharp $H_{p}-L_{p}$ inequality reads as follows. 


\section{Theorem 1}

(a) Let $f \in H_{p}$, where $0<p<1 /(1+\alpha)$ for some $0<\alpha \leq 1$, and $\left\{q_{k}: k \in \mathbb{N}\right\}$ be a sequence of non-increasing numbers satisfying conditions (11) and (12). Then the maximal operator

$$
\widetilde{t}_{p, \alpha}^{*}:=\sup _{n \in \mathbb{N}} \frac{\left|t_{n} f\right|}{(n+1)^{1 / p-1-\alpha}}
$$

is bounded from the martingale Hardy space $H_{p}$ to the Lebesgue space $L_{p}$, i.e. the following inequality holds:

$$
\left\|\sup _{n \in \mathbb{N}}\left|t_{n} f\right| /\left((n+1)^{1 / p-1-\alpha}\right)\right\|_{p} \leq c_{\alpha, p}\|f\|_{H_{p}} .
$$

(b) Let $0<p<1 /(1+\alpha)$ for some $0<\alpha \leq 1$, and $\left\{\Phi_{n}: n \in \mathbb{N}_{+}\right\}$be any non-decreasing sequence, satisfying the condition

$$
\varlimsup_{n \rightarrow \infty} \frac{(n+1)^{1 / p-1-\alpha}}{\Phi_{n}}=\infty .
$$

Then the inequality (15) is sharp in the sense that there exist a Nörlund mean with non-increasing sequence $\left\{q_{k}: k \in \mathbb{N}\right\}$ satisfying the conditions (11) and (12) and a martingale $f \in H_{P}$ such that

$$
\sup _{k \in \mathbb{N}} \frac{\left\|\frac{t_{M_{2 n_{k}}+1} f_{k}}{\Phi_{M_{2 n_{k}+1}+1}}\right\|_{\text {weak- } L_{p}}}{\left\|f_{k}\right\|_{H_{p}}}=\infty .
$$

Our new result concerning strong summability of Nörlund means with non-increasing sequences reads as follows.

Theorem 2 Let $f \in H_{p}$, where $0<\alpha<1,0<p<1 /(1+\alpha)$, and let $\left\{q_{n}: n \geq 0\right\}$ be a sequence of non-increasing numbers, satisfying conditions (11) and (12). Then there exists an absolute constant $c_{\alpha, p}$, depending only on $\alpha$ and $p$, such that the inequality

$$
\sum_{k=1}^{\infty} \frac{\left\|t_{k} f\right\|_{p}^{p}}{k^{2-(1+\alpha) p}} \leq c_{\alpha, p}\|f\|_{H_{p}}^{p}
$$

holds.

\section{Lemmas}

We need the following well-known lemma of Weisz [34].

Lemma 1 Suppose that an operator $T$ is $\sigma$-linear and for some $0<p \leq 1$ and

$$
\int_{\bar{I}}|T a|^{p} d \mu \leq c_{p}<\infty
$$

for every p-atom a, where I denotes the support of the atom. If $T$ is bounded from $L_{\infty}$ to $L_{\infty}$, then

$$
\|T f\|_{p} \leq c_{p}\|f\|_{H_{p}} .
$$


The next results are due to Blahota, Persson, and Tephnadze [27].

Lemma 2 Let $s_{n} M_{n}<r \leq\left(s_{n}+1\right) M_{n}$, where $1 \leq s_{n} \leq m_{n}-1$. Then for every Nörlund mean, without any restriction on the generative sequence $\left\{q_{k}: k \in \mathbb{N}\right\}$ we have the following equality:

$$
\begin{aligned}
Q_{r} F_{r}= & Q_{r} D_{s_{n} M_{n}}-\psi_{s_{n} M_{n}-1} \sum_{l=1}^{s_{n} M_{n}-2}\left(q_{r-s_{n} M_{n}+l}-q_{r-s_{n} M_{n}+l+1}\right) l \overline{K_{l}} \\
& -\psi_{s_{n} M_{n}-1}\left(s_{n} M_{n}-1\right) q_{r-1} \overline{K_{s_{n} M_{n}-1}}+\psi_{s_{n} M_{n}} Q_{r-s_{n} M_{n}} F_{r-s_{n} M_{n}} .
\end{aligned}
$$

We also need the following new lemmas of independent interest.

Lemma 3 Let $0<\alpha \leq 1$ and $\left\{q_{n}: n \geq 0\right\}$ be a sequence of non-increasing numbers satisfying conditions (11) and (12). Then

$$
\left|Q_{n} F_{n}\right| \leq c_{\alpha}\left\{\sum_{j=0}^{|n|} M_{j}^{\alpha} \varphi_{j}\left|K_{M_{j}}\right|\right\}
$$

where

$$
\varlimsup_{j \rightarrow \infty} \frac{\varphi_{j}}{j^{\varepsilon}}=0, \quad \text { for every } \varepsilon>0
$$

Lemma 4 Let $0<\alpha \leq 1$ and $\left\{q_{n}: n \geq 0\right\}$ be a sequence of non-increasing numbers, satisfying conditions (11) and (12). If $r \geq M_{N}$, then

$$
\int_{I_{N}}\left|F_{r}(x-t)\right| d \mu(t) \leq \frac{c_{\alpha} M_{l}^{\alpha} \varphi_{l} M_{k}}{r^{\alpha} M_{N}} \leq \frac{c_{\alpha} M_{l}^{\alpha} \varphi_{l} M_{k}}{M_{N}^{1+\alpha}}, \quad x \in I_{l+1}\left(s_{k} e_{k}+s_{l} e_{l}\right),
$$

where

$$
1 \leq s_{k} \leq m_{k}-1, \quad 1 \leq s_{l} \leq m_{l}-1 \quad(k=0, \ldots, N-2, l=k+1, \ldots, N-1)
$$

and

$$
\int_{I_{N}}\left|F_{r}(x-t)\right| d \mu(t) \leq \frac{c_{\alpha} M_{k}}{M_{N}}, \quad x \in I_{N}\left(s_{k} e_{k}\right)
$$

where

$$
1 \leq s_{k} \leq m_{k}-1 \quad(k=0, \ldots, N-1)
$$

\section{Proofs}

Proof of Lemma 3 Let $0<\alpha \leq 1$ and $\left\{q_{k}: k \geq 0\right\}$ satisfy the conditions (11) and (12). Since

$$
n^{\alpha} \varphi_{n} \geq Q_{n} \geq n q_{n-1}
$$


we obtain

$$
q_{n-1} \leq n^{\alpha-1} \varphi_{n}
$$

where $\varphi_{n}$ satisfies condition (17).

By using an Abel transformation we get

$$
Q_{n}=\sum_{j=1}^{n-2}\left(q_{j}-q_{j+1}\right) j+q_{n-1}(n-1)+q_{0}
$$

and

$$
\sum_{j=1}^{n-2}\left|q_{j}-q_{j+1}\right| j \leq Q_{n} \leq n^{\alpha} \varphi_{n}
$$

Suppose that

$$
\left|q_{j}-q_{j+1}\right| \geq j^{\alpha-2} \phi_{j} \delta_{j}
$$

for all $j \in \mathbb{N}$, where $\delta_{j}$ is any function, such that

$$
\varlimsup_{j \rightarrow \infty} \delta_{j}=\infty
$$

Under condition (19) there exists an increasing sequence $\left\{\alpha_{k}: k \geq 0\right\}$, such that $\alpha_{k+1} \geq$ $2 \alpha_{k}$ and

$$
\delta_{\alpha_{k}} \uparrow \infty .
$$

Hence,

$$
\begin{aligned}
\sum_{j=\alpha_{k}}^{\alpha_{k+1}+1}\left|q_{j}-q_{j+1}\right| j & \geq c \phi_{\alpha_{k}} \delta_{\alpha_{k}} \sum_{j=\alpha_{k}}^{\alpha_{k+1}+1} j^{\alpha-1} \\
& \geq c \phi_{\alpha_{k}} \delta_{\alpha_{k}} \int_{\alpha_{k}}^{\alpha_{k+1}+2} x^{\alpha-1} d x \\
& \geq\left.\frac{c \phi_{\alpha_{k}} \delta_{\alpha_{k}}}{\alpha} x^{\alpha}\right|_{\alpha_{k}} ^{\alpha_{k+1}} \geq c \phi_{\alpha_{k}} \delta_{\alpha_{k}} \alpha_{k}^{\alpha} .
\end{aligned}
$$

By combining (18) and (22) we get

$$
\frac{Q_{\alpha_{k+1}+3}}{\left(\alpha_{k+1}+3\right)^{\alpha}\left(\phi_{\alpha_{k+1}}+3\right)} \geq \frac{\sum_{j=1}^{\alpha_{k+1}+1}\left|q_{j}-q_{j+1}\right| j}{\left(\alpha_{k+1}+3\right)^{\alpha}\left(\phi_{\alpha_{k+1}}+3\right)} \geq c \delta_{\alpha_{k}} \rightarrow \infty, \quad \text { as } k \rightarrow \infty .
$$

This is a contradiction with condition (13), that is,

$$
\frac{Q_{n}}{n^{\alpha} \phi_{n}}=O(1), \quad \text { as } n \rightarrow \infty \text {. }
$$


It follows that

$$
\left|q_{j}-q_{j+1}\right| \leq j^{\alpha-2} \phi_{j}
$$

It is easy to see that

$$
Q_{k}\left|D_{s M_{n}}\right| \leq c M_{n}^{\alpha} \phi_{n}\left|D_{s M_{n}}\right|
$$

and

$$
\left(s M_{n}-1\right) q_{k-1}\left|K_{s M_{n}-1}\right| \leq c \phi_{k} k^{\alpha-1} M_{n}\left|K_{s M_{n}-1}\right| \leq c M_{n}^{\alpha} \phi_{n}\left|K_{s M_{n}-1}\right| .
$$

Let

$$
n=s_{n_{1}} M_{n_{1}}+s_{n_{2}} M_{n_{2}}+\cdots+s_{n_{r}} M_{n_{r}}, \quad n_{1}>n_{2}>\cdots>n_{r},
$$

and

$$
n^{(k)}=s_{n_{k+1}} M_{n_{k+1}}+\cdots+s_{n_{r}} M_{n_{r}}, \quad 1 \leq s_{n_{l}} \leq m_{l}-1, l=1, \ldots, r .
$$

By combining (24)-(26) and Lemma 2 we have

$$
\begin{aligned}
& \left|Q_{n} F_{n}\right| \\
& \leq c_{\alpha} M_{n_{1}}^{\alpha} \phi_{n_{1}}\left|D_{s_{n_{1}} M_{n_{1}}}\right|+c_{\alpha} \sum_{l=1}^{s_{n_{1}} M_{n_{1}}-1}\left|\left(n^{(1)}+l\right)^{\alpha-2}\right| \phi_{n^{(1)}+l}\left|l K_{l}\right| \\
& +c_{\alpha} M_{n_{1}}^{\alpha} \phi_{n_{1}}\left|K_{s_{n_{1}} M_{n_{1}}-1}\right|+c_{\alpha}\left|Q_{n^{(1)}} F_{n^{(1)}}\right| .
\end{aligned}
$$

By repeating this process $r$ times we get

$$
\begin{aligned}
& \left|Q_{n} F_{n}\right| \\
& \quad \leq c_{\alpha} \sum_{k=1}^{r}\left(M_{n_{k}}^{\alpha} \phi_{n_{k}}\left|D_{s_{n_{k}} M_{n_{k}}}\right|+\sum_{l=1}^{s_{n_{k}} M_{n_{k}}-1}\left(n^{(k)}+l\right)^{\alpha-2} \phi_{n^{(k)}+l}\left|l K_{l}\right|+M_{n_{k}}^{\alpha} \phi_{n_{k}}\left|K_{s_{n_{k}} M_{n_{k}}-1}\right|\right) \\
& \quad:=I+I I+I I I .
\end{aligned}
$$

By combining (8), (9), and (14) we find that

$$
I \leq c_{\alpha} \sum_{k=1}^{|n|} M_{k}^{\alpha} \phi_{k}\left|D_{M_{k}}\right| \leq c_{\alpha} \sum_{k=1}^{|n|} M_{k}^{\alpha} \phi_{k}\left|K_{M_{k}}\right|
$$

and

$$
I I I \leq c_{\alpha} \sum_{k=1}^{r} M_{n_{k}}^{\alpha-1} \phi_{n_{k}}\left|M_{n_{k}} K_{s_{n_{k}} M_{n_{k}}}-D_{s_{n_{k}} M_{n_{k}}}\right| \leq c_{\alpha} \sum_{k=1}^{r} M_{k}^{\alpha} \phi_{n_{k}}\left|K_{M_{k}}\right| .
$$


Moreover,

$$
\begin{aligned}
I I= & c_{\alpha} \sum_{k=1}^{r} \sum_{A=1}^{n_{k}} \sum_{l=s_{A-1} M_{A-1}}^{s_{A} M_{A}-1}\left(n^{(k)}+l\right)^{\alpha-2} \phi_{n^{(k)}+l}\left|l K_{l}\right| \\
= & c_{\alpha} \sum_{k=1}^{r} \sum_{A=1}^{n_{k+1}} \sum_{l=s_{A-1} M_{A-1}}^{s_{A} M_{A}-1}\left(n^{(k)}+l\right)^{\alpha-2} \phi_{n^{(k)}+l}\left|l K_{l}\right| \\
& +c_{\alpha} \sum_{k=1}^{r} \sum_{A=n_{k+1}+1 l=s_{A-1} M_{A-1}}^{n_{k}} \sum_{s_{A} M_{A}-1}\left(n^{(k)}+l\right)^{\alpha-2} \phi_{n^{(k)}+l}\left|l K_{l}\right| \\
\leq & c_{\alpha} \sum_{k=1}^{r} M_{n_{k+1}}^{\alpha-2} \phi_{n_{k+1}} \sum_{A=1}^{n_{k+1}} \sum_{l=s_{A-1} M_{A-1} M_{A}-1}^{s_{A}}\left|l K_{l}\right| \\
& +c_{\alpha} \sum_{k=1}^{r} \sum_{A=n_{k+1}+1}^{n_{k}} M_{A}^{\alpha-2} \phi_{A} \sum_{l=s_{A-1} M_{A-1}}^{s_{A}-1}\left|l K_{l}\right|:=I I_{1}+I I_{2} .
\end{aligned}
$$

By applying (14) for $I I_{1}$ we get

$$
\begin{aligned}
I I_{1} & \leq c_{\alpha} \sum_{k=1}^{r} M_{n_{k+1}}^{\alpha-2} \phi_{n_{k+1}} \sum_{A=1}^{n_{k+1}} \sum_{l=s_{A-1} M_{A-1}}^{s_{A} M_{A}-1} \sum_{j=0}^{A} M_{j}\left|K_{M_{j}}\right| \\
& \leq c_{\alpha} \sum_{k=1}^{n_{1}} M_{k}^{\alpha-2} \phi_{k} \sum_{A=1}^{k} M_{A} \sum_{j=0}^{A} M_{j}\left|K_{M_{j}}\right| \\
& \leq c_{\alpha} \sum_{k=0}^{n_{1}} M_{k}^{\alpha-1} \phi_{k} \sum_{j=0}^{k} M_{j}\left|K_{M_{j}}\right| \\
& =c_{\alpha} \sum_{j=0}^{n_{1}} M_{j}\left|K_{M_{j}}\right| \sum_{k=j}^{n_{1}} \phi_{k} M_{k}^{\alpha-1} \leq c_{\alpha} \sum_{j=0}^{n_{1}} \phi_{j} M_{j}^{\alpha}\left|K_{M_{j}}\right| .
\end{aligned}
$$

By using (14) for $\mathrm{II}_{2}$ we have similarly

$$
\begin{aligned}
I I_{2} & \leq c_{\alpha} \sum_{k=1}^{r} \sum_{A=n_{k+1}+1}^{n_{k}} M_{A}^{\alpha-1} \phi_{A} \sum_{j=0}^{A} M_{j}\left|K_{M_{j}}\right| \\
& \leq c_{\alpha} \sum_{A=1}^{n_{1}} M_{A}^{\alpha-1} \phi_{A} \sum_{j=0}^{A} M_{j}\left|K_{M_{j}}\right| \leq c_{\alpha} \sum_{j=0}^{n_{1}} \phi_{j} M_{j}^{\alpha}\left|K_{M_{j}}\right| .
\end{aligned}
$$

The proof is complete by combining the estimates above.

Proof ofLemma 4 Let $x \in I_{l+1}\left(s_{k} e_{k}+s_{l} e_{l}\right), 1 \leq s_{k} \leq m_{k}-1,1 \leq s_{l} \leq m_{l}-1$. Then, by applying (14), we have

$$
K_{M_{n}}(x)=0, \quad \text { when } n>l>k \text {. }
$$


Suppose that $k<n \leq l$. Moreover, by using (14) we get

$$
\left|K_{M_{n}}(x)\right| \leq c M_{k}
$$

Let $n \leq k<l$. Then

$$
\left|K_{M_{n}}(x)\right|=\left(M_{n}+1\right) / 2 \leq c M_{k} .
$$

If we now apply Lemma 3 and (14) we can conclude that

$$
Q_{r}\left|F_{r}(x)\right| \leq c_{\alpha} \sum_{A=0}^{l} M_{A}^{\alpha} \varphi_{A}\left|K_{M_{A}}(x)\right| \leq c_{\alpha} \sum_{A=0}^{l} M_{A}^{\alpha} \varphi_{A} M_{k} \leq c_{\alpha} M_{l}^{\alpha} \varphi_{l} M_{k} .
$$

Let $x \in I_{l+1}\left(s_{k} e_{k}+s_{l} e_{l}\right)$, for some $0 \leq k<l \leq N-1$. Since $x-t \in I_{l+1}\left(s_{k} e_{k}+s_{l} e_{l}\right)$, for $t \in I_{N}$ and $r \geq M_{N}$ from (27) we obtain

$$
\int_{I_{N}}\left|F_{r}(x-t)\right| d \mu(t) \leq \frac{c_{\alpha} M_{l}^{\alpha} \varphi_{l} M_{k}}{r^{\alpha} M_{N}}
$$

Let $x \in I_{N}\left(s_{k} e_{k}\right), k=0, \ldots, N-1$. Then, by applying (8) and (9) we have

$$
\begin{aligned}
\int_{I_{N}}\left|F_{r}(x-t)\right| d \mu(t) & \leq \frac{1}{Q_{n}} \sum_{m=1}^{n} q_{n-m} \int_{I_{N}}\left|D_{m}(x-t)\right| d \mu(t) \\
& \leq \frac{1}{Q_{n}} \sum_{m=1}^{n} q_{n-m} \frac{c M_{k}}{M_{N}} \leq \frac{c M_{k}}{M_{N}} .
\end{aligned}
$$

By combining (28) and (29) we complete the proof of Lemma 4.

Proof of Theorem 1 According to Lemma 1 the proof of the first part of Theorem 1 will be complete, if we show that

$$
\int_{\bar{I}_{N}}\left|\stackrel{\sim}{t}, p^{*} a(x)\right|^{p} d \mu(x)<\infty
$$

for every $1 /(1+\alpha-\varepsilon)$-atom $a$. We may assume that $a$ is an arbitrary $p$-atom with support $I, \mu(I)=M_{N}^{-1}$ and $I=I_{N}$. It is easy to see that $t_{n}(a)=0$, when $n \leq M_{N}$. Therefore, we can suppose that $n>M_{N}$.

By using Lemma 3 we easily see that $\widetilde{\sim}^{*, p}$ is bounded from $L_{\infty}$ to $L_{\infty}$. Let $x \in I_{N}$. Since $\|a\|_{\infty} \leq M_{N}^{1 / p}$ we obtain

$$
\begin{aligned}
\left|t_{n} a(x)\right| & \leq \int_{I_{N}}|a(t)|\left|F_{n}(x-t)\right| d \mu(t) \\
& \leq\|a\|_{\infty} \int_{I_{N}}\left|F_{n}(x-t)\right| d \mu(t) \leq M_{N}^{1 / p} \int_{I_{N}}\left|F_{n}(x-t)\right| d \mu(t) .
\end{aligned}
$$

Let $x \in I_{l+1}\left(s_{k} e_{k}+s_{l} e_{l}\right), 0 \leq k<l<N$. From Lemma 4 we get

$$
\left|t_{n} a(x)\right| \leq \frac{c_{\alpha} M_{l}^{\alpha} \varphi_{l} M_{k} M_{N}^{1 / p}}{n^{\alpha} M_{N}} \leq \frac{c_{\alpha} \varphi_{l} M_{l}^{\alpha} M_{k}}{n^{\alpha} M_{N}^{1-1 / p}} \leq \frac{c_{\alpha} \varphi_{l} M_{l}^{\alpha} M_{k}}{M_{N}^{1+\alpha-1 / p}} .
$$


Let $x \in I_{N}\left(s_{k} e_{k}\right), 0 \leq k<N$. According to Lemma 4 we have

$$
\left|t_{n} a(x)\right| \leq \frac{c_{\alpha} M_{N}^{1 / p} M_{k}}{M_{N}} \leq c_{\alpha} M_{N}^{1 / p-1} M_{k}
$$

By combining (7) and (30)-(31) we obtain

$$
\begin{aligned}
\int_{I_{N}}\left|t^{*, p} a\right|^{p} d \mu \\
=\sum_{k=0}^{N-2} \sum_{s_{k}=1}^{m_{k}-1} \sum_{l=k+1}^{N-1} \sum_{s_{l}=1}^{m_{l}-1} \int_{I_{l+1}\left(s_{k} e_{k}+s_{l} e_{l}\right)} \sup _{n>M_{N}}\left|\frac{t_{n} a}{n^{1 / p-1-\alpha}}\right|^{p} d \mu \\
\quad+\sum_{k=0}^{N-1} \sum_{s_{k}=1}^{m_{k}-1} \int_{I_{N}\left(s_{k} e_{k}\right)} \sup _{n>M_{N}}\left|\frac{t_{n} a}{n^{1 / p-1-\alpha}}\right|^{p} d \mu \\
\leq c_{\alpha} \sum_{k=0}^{N-2} \sum_{l=k+1}^{N-1} \frac{\left(m_{k}-1\right)\left(m_{l}-1\right)}{M_{l+1}}\left(\varphi_{l} M_{l}^{\alpha} M_{k}\right)^{p}+c_{\alpha} \sum_{k=0}^{N-1} \frac{\left(m_{k}-1\right)}{M_{N}} M_{N}^{\alpha p} M_{k}^{p} \\
\leq c_{\alpha} \sum_{k=0}^{N-2} \sum_{l=k+1}^{N-1} \frac{\left(\varphi_{l} M_{l}^{\alpha} M_{k}\right)^{p}}{M_{l+1}}+c_{\alpha} \sum_{k=0}^{N-1} \frac{M_{k}^{p}}{M_{N}^{(1-\alpha p)}} \\
\leq c_{\alpha} \sum_{k=0}^{N-2} M_{k}^{p} \sum_{l=k+1}^{N-1} \frac{\varphi_{l}^{p}}{M_{l}^{1-\alpha p}}+c_{\alpha} \leq \sum_{k=0}^{N-2} M_{k}^{p} \frac{\varphi_{k}^{p}}{M_{k}^{1-\alpha p}}+c_{\alpha} \\
\leq c_{\alpha} \sum_{k=0}^{N-2} \frac{\varphi_{k}^{p}}{M_{k}^{1-p(\alpha+1)}+c_{\alpha} \leq c_{\alpha} \sum_{k=0}^{p-2} \frac{\varphi_{k}^{p}}{2^{k(1-p(1+\alpha))}}+c_{\alpha} \leq c_{\alpha}<\infty}
\end{aligned}
$$

The proof of part (a) is complete.

Under condition (16) there exist positive integers $n_{k}$ such that

$$
\lim _{k \rightarrow \infty} \frac{\left(M_{2 n_{k}}+1\right)^{1 / p-1-\alpha}}{\Phi_{M_{2 n_{k}}+1}}=\infty, \quad 0<p<1 /(1+\alpha)
$$

Let $t_{n}$ be Nörlund mean with non-increasing sequence $\left\{q_{k}: k \in \mathbb{N}\right\}$ satisfying (11) and condition (12), but in the restricted form

$$
\frac{c_{\alpha}\left(M_{2 n_{k}}+1\right)^{1 / p-1-\alpha}}{\varphi_{M_{2 n_{k}}+1} \Phi_{M_{2 n_{k}}+1}} \rightarrow \infty, \quad \text { as } k \rightarrow \infty
$$

Set

$$
f_{k}:=D_{M_{2 n_{k}+1}}-D_{M_{2 n_{k}}} \text {. }
$$

Then

$$
\widehat{f}_{k}(i)= \begin{cases}1, & i=M_{2 n_{k}}, \ldots, M_{2 n_{k}+1}-1 \\ 0, & \text { otherwise }\end{cases}
$$


and

$$
S_{i} f_{k}= \begin{cases}D_{i}-D_{M_{2 n_{k}}}, & i=M_{2 n_{k}}+1, \ldots, M_{2 n_{k}+1}-1, \\ f_{k}, & i \geq M_{2 n_{k}+1}, \\ 0, & \text { otherwise. }\end{cases}
$$

Moreover,

$$
\left\|f_{k}\right\|_{H_{p}} \leq \lambda M_{2 n_{k}}^{1-1 / p}
$$

where $\lambda=\sup _{n} m_{n}$.

By using (33) we get

$$
\begin{aligned}
\frac{\left|t_{M_{2 n_{k}+1}} f_{k}\right|}{\Phi_{M_{2 n_{k}}+1}} & =\frac{q_{0}\left|S_{M_{2 n_{k}}+1}\right|}{Q_{M_{2 n_{k}}+1} \Phi_{M_{2 n_{k}}+1}} \\
& =\frac{q_{0}\left|D_{M_{2 n_{k}}+1}-D_{M_{2 n_{k}}}\right|}{Q_{M_{2 n_{k}}+1} \Phi_{M_{2 n_{k}}+1}}=\frac{q_{0}\left|\psi_{M_{2 n_{k}}}\right|}{Q_{M_{2 n_{k}}+1} \Phi_{M_{2 n_{k}}+1}} \geq \frac{c_{\alpha}}{M_{2 n_{k}}^{\alpha} \Phi_{M_{2 n_{k}}+1} \varphi_{M_{2 n_{k}}+1}} .
\end{aligned}
$$

Hence,

$$
\mu\left\{x \in G_{m}: \frac{\left|t_{M_{2 n_{k}}+1} f_{k}(x)\right|}{\Phi_{M_{2 n_{k}}+1}} \geq \frac{c_{\alpha}}{M_{2 n_{k}}^{\alpha} \Phi_{M_{2 n_{k}}+1} \varphi_{M_{2 n_{k}}+1}}\right\}=1 .
$$

By applying (35) we have

$$
\begin{gathered}
\frac{\frac{c_{\alpha}}{M_{2 n_{k}}^{\alpha} \Phi_{M_{2 n_{k}}+1 \varphi_{M_{2 n_{k}}+1}}}\left(\mu \left\{x \in G_{m}: \frac{\mid t_{M_{2 n_{k}+1}+f_{k}(x) \mid}}{\Phi_{M_{2 n_{k}}+1}} \geq \frac{q_{0}}{\left.\left.M_{2 n_{k}}^{\alpha} \Phi_{M_{2 n_{k}}+1 \varphi_{M_{2 n_{k}}+1}}\right\}\right)^{1 / p}}\right.\right.}{\left\|f_{k}\right\|_{H_{p}}} \\
\geq \frac{c_{\alpha} M_{2 n_{k}}^{1 / p-1-\alpha}}{\Phi_{M_{2 n_{k}}+1} \varphi_{M_{2 n_{k}}+1}} \geq \frac{c_{\alpha}\left(M_{2 n_{k}}+1\right)^{1 / p-1-\alpha}}{\Phi_{M_{2 n_{k}}+1} \varphi_{M_{2 n_{k}}+1}} \rightarrow \infty, \quad \text { as } k \rightarrow \infty .
\end{gathered}
$$

The proof is complete.

Proof of Theorem 2 According to Lemma 1 the proof of Theorem 2 will be complete, if we show that

$$
\sum_{m=1}^{\infty} \frac{\left\|t_{m} a\right\|_{p}^{p}}{m^{2-(1+\alpha) p}} \leq c_{\alpha}<\infty
$$

for every $p$-atom $a$. Analogously to the first part of Theorem 1 we can assume that $n>M_{N}$ and $a$ be an arbitrary $p$-atom, with support $I, \mu(I)=M_{N}$, and $I=I_{N}$.

Let $x \in I_{N}$. Since $\|a\|_{\infty} \leq c M_{N}^{1 / p}$ if we apply Lemma 3 we obtain

$$
\int_{I_{N}}\left|t_{m} a\right|^{p} d \mu \leq \int_{I_{N}}\left\|F_{m}\right\|_{1}^{p}\|a\|_{\infty}^{p} d \mu \leq c_{\alpha, p} \varphi_{m}^{p} \int_{I_{N}}\|a\|_{\infty}^{p} d \mu \leq c_{\alpha, p} \varphi_{m}^{p} .
$$

Hence

$$
\sum_{m=M_{N}+1}^{\infty} \frac{\int_{I_{N}}\left|t_{m} a\right|^{p} d \mu}{m^{2-(1+\alpha) p}} \leq c_{\alpha, p} \sum_{m=M_{N}+1}^{\infty} \frac{\varphi_{m}^{p}}{m^{2-(1+\alpha) p}} \leq \frac{c_{\alpha, p} \varphi_{N}^{p}}{2^{N(1-(1+\alpha) p)}} \leq c_{\alpha, p}<\infty
$$


By combining (7), (30), and (31) analogously to first part of Theorem 1 we can write

$$
\begin{aligned}
& \sum_{m=M_{N}+1}^{\infty} \frac{\int_{I_{N}}\left|t_{m} a\right|^{p} d \mu}{m^{2-(1+\alpha) p}} \\
& =\sum_{m=M_{N}+1}^{\infty}\left(\sum_{k=0}^{N-2} \sum_{s_{k}=1}^{m_{k}-1} \sum_{l=k+1}^{N-1} \sum_{s_{l}=1}^{m_{l}-1} \frac{\int_{I_{l+1}\left(s_{k} e_{k}+s_{l} e_{l}\right)}\left|t_{m} a\right|^{p} d \mu}{m^{2-(1+\alpha) p}}+\sum_{k=0}^{N-1} \sum_{s_{k}=0}^{m_{k}-1} \frac{\int_{I_{N}\left(s_{k} e_{k}\right)}\left|t_{m} a\right|^{p} d \mu}{m^{2-(1+\alpha) p}}\right) \\
& \leq \sum_{m=M_{N}+1}^{\infty}\left(\frac{c_{\alpha, p} M_{N}^{1-p}}{m^{2-p}} \sum_{k=0}^{N-2} \sum_{l=k+1}^{N-1} \frac{M_{l}^{p \alpha} \varphi_{l}^{p} M_{k}^{p}}{M_{l}}+\frac{c_{\alpha, p} M_{N}^{1-p}}{m^{2-(1+\alpha) p}} \sum_{k=0}^{N-1} \frac{M_{k}^{p}}{M_{N}}\right) \\
& <c_{\alpha, p} M_{N}^{1-p} \sum_{m=M_{N}+1}^{\infty} \frac{1}{m^{2-p}}+c_{\alpha, p} \sum_{m=M_{N}+1}^{\infty} \frac{1}{m^{2-(1+\alpha) p}} \leq c_{\alpha, p}<\infty,
\end{aligned}
$$

which completes the proof.

\section{Applications and final remarks}

Remark 1 We note that under the conditions (3) and (4) we see that the conditions (11) and (12) are also fulfilled.

Proof Let $0<\alpha \leq 1$. We can write

$$
\left|Q_{n}\right|=\left|\sum_{j=1}^{n-2}\left(q_{j}-q_{j+1}\right) j+q_{n-1}(n-1)+q_{0}\right| .
$$

First suppose that $\alpha=1$. Then

$$
q_{n-1} \leq q_{0} \leq c
$$

and

$$
\left|Q_{n}\right| \leq\left|\sum_{j=1}^{n-2} j^{0}+c(n-1)+q_{0}\right| \leq c n .
$$

Moreover, condition (12) automatically holds,

$$
\frac{Q_{n}}{n^{1+\varepsilon}} \leq \frac{q_{0} n}{n^{1+\varepsilon}} \rightarrow 0, \quad \text { as } n \rightarrow \infty,
$$

for any $\varepsilon>0$.

Since the case $q_{0} n / Q_{n}=O(1)$, as $n \rightarrow \infty$, has already been considered, we can exclude it. Hence, we may assume that $\left\{q_{k}: k \geq 0\right\}$ satisfies conditions (3) and (4) and, in addition, satisfies the following condition:

$$
\frac{Q_{n}}{q_{0} n}=o(1), \quad \text { as } n \rightarrow \infty \text {. }
$$

It follows that

$$
q_{n}=q_{0} \frac{q_{n} n}{q_{0} n} \leq q_{0} \frac{Q_{n}}{q_{0} n}=o(1), \quad \text { as } n \rightarrow \infty,
$$




$$
q_{n}=\sum_{l=n}^{\infty}\left(q_{l}-q_{l+1}\right) \leq \sum_{l=n}^{\infty} \frac{1}{l^{2-\alpha}} \leq \frac{c}{n^{1-\alpha}},
$$

and

$$
\left|Q_{n}\right| \leq\left|\sum_{j=1}^{n-2} j^{\alpha-1}+c(n-1)^{\alpha}+q_{0}\right| \leq c n^{\alpha} .
$$

From Remark 1 we immediately see that the following is true.

Corollary 1 Conditions (3) and (4) provide a wider class of Nörlund means with nonincreasing coefficients than conditions (11) and (12).

From the proof of Remark 1 for $\alpha=1$ we immediately have the following.

Remark 2 Let $\alpha=1$ and $\left\{q_{k}: k \in \mathbb{N}\right\}$ be a sequence of non-increasing numbers. Then condition (12) automatically holds,

$$
\frac{Q_{n}}{n^{1+\varepsilon}} \rightarrow 0, \quad \text { as } n \rightarrow \infty
$$

for any $\varepsilon>0$.

By applying Remark 2 and Theorem 1 we get the following.

\section{Theorem 3}

(a) Let $f \in H_{p}$, where $0<p<1 / 2$ and $\left\{q_{k}: k \in \mathbb{N}\right\}$ be a sequence of non-increasing numbers satisfying condition (11) for $\alpha=1$. Then the maximal operator

$$
\tilde{t}_{p, 1}^{*}:=\sup _{n \in \mathbb{N}} \frac{\left|t_{n} f\right|}{(n+1)^{1 / p-2}}
$$

is bounded from the martingale Hardy space $H_{p}$ to the Lebesgue space $L_{p}$, i.e. the inequality

$$
\left\|\sup _{n \in \mathbb{N}}\left|t_{n} f\right| /\left((n+1)^{1 / p-2}\right)\right\|_{p} \leq c_{\alpha, p}\|f\|_{H_{p}}
$$

holds.

(b) Let $\left\{\Phi_{n}: n \in \mathbb{N}_{+}\right\}$be any non-decreasing sequence, satisfying the condition

$$
\varlimsup_{n \rightarrow \infty} \frac{(n+1)^{1 / p-2}}{\Phi_{n}}=\infty .
$$

Then the inequality (36) is sharp in the sense that there exists a Nörlund mean with non-increasing sequence $\left\{q_{k}: k \in \mathbb{N}\right\}$ satisfying the condition (11) such that

$$
\sup _{k \in \mathbb{N}} \frac{\left\|\frac{t_{M_{2 n_{k}}+1} f_{k}}{\Phi_{M_{2 n_{k}}+1}}\right\|_{\text {weak- } L_{p}}}{\left\|f_{k}\right\|_{H_{p}}}=\infty .
$$


By applying Remark 2 and Theorem 2 we get the following.

Theorem 4 Let $f \in H_{p}$, where $0<p<1 / 2$ and $\left\{q_{n}: n \geq 0\right\}$ be a sequence of non-increasing numbers, satisfying condition (11). Then there exists an absolute constant $c_{\alpha, p}$, depending only on $\alpha$ and $p$, such that

$$
\sum_{k=1}^{\infty} \frac{\left\|t_{k} f\right\|_{H_{p}}^{p}}{k^{2-(1+\alpha) p}} \leq c_{\alpha, p}\|f\|_{H_{p}}^{p} .
$$

From Theorem 3 we get the following result by Tephnadze [35].

\section{Corollary 2}

(a) Let $f \in H_{p}$, where $0<p<1 / 2$. Then the maximal operator

$$
\tilde{\sigma}_{p}^{*}:=\sup _{n \in \mathbb{N}} \frac{\left|\sigma_{n} f\right|}{(n+1)^{1 / p-2}}
$$

is bounded from the martingale Hardy space $H_{p}$ to the Lebesgue space $L_{p}$.

(b) Let $\left\{\Phi_{n}: n \in \mathbb{N}_{+}\right\}$be any non-decreasing sequence, satisfying the condition

$$
\varlimsup_{n \rightarrow \infty} \frac{(n+1)^{1 / p-2}}{\Phi_{n}}=\infty .
$$

Then

$$
\sup _{k \in \mathbb{N}} \frac{\left\|\frac{\sigma_{M_{2 n_{k}}+1} f_{k}}{\Phi_{M_{2 n_{k}}+1}}\right\|_{\text {weak }-L_{p}}}{\left\|f_{k}\right\|_{H_{p}}}=\infty .
$$

Moreover, Theorem 4 implies the following result by Tephnadze [18].

Corollary 3 Let $f \in H_{p}$, where $0<p<1 / 2$. Then there exists an absolute constant $c_{p}$, depending only on $p$, such that

$$
\sum_{k=1}^{\infty} \frac{\left\|\sigma_{k} f\right\|_{p}^{p}}{k^{2-2 p}} \leq c_{p}\|f\|_{H_{p}}^{p} .
$$

Next we note that Theorem 1 and Remark 1 imply the following results of Blahota, Tephnadze [13] for $0<\alpha<1$.

\section{Corollary 4}

(a) Let $f \in H_{p}$, where $0<p<1 /(1+\alpha)$ for some $0<\alpha<1$. Then the maximal operator

$$
\widetilde{\sigma}_{p}^{*, \alpha}:=\sup _{n \in \mathbb{N}} \frac{\left|\sigma_{n}^{\alpha} f\right|}{(n+1)^{1 / p-1-\alpha}}
$$

is bounded from the martingale Hardy space $H_{p}$ to the Lebesgue space $L_{p}$.

(b) Let $\left\{\Phi_{n}: n \in \mathbb{N}_{+}\right\}$be any non-decreasing sequence, satisfying the condition

$$
\varlimsup_{n \rightarrow \infty} \frac{(n+1)^{1 / p-1-\alpha}}{\Phi_{n}}=\infty .
$$


Then

$$
\sup _{k \in \mathbb{N}} \frac{\left\|\frac{\sigma_{M_{2 n_{k}+1}}^{\alpha} f_{k}}{\Phi_{M_{2 n_{k}+1}}}\right\|_{\text {weak }-L_{p}}}{\left\|f_{k}\right\|_{H_{p}}}=\infty .
$$

Similarly, Theorem 2 and Remark 1 immediately imply the following result of Blahota, Tephnadze [13] for $0<\alpha<1$.

Corollary 5 Let $f \in H_{p}$, where $0<p<1 /(1+\alpha)$, for some $0<\alpha<1$. Then there exists an absolute constant $c_{\alpha, p}$, depending only on $\alpha$ and $p$, such that

$$
\sum_{k=1}^{\infty} \frac{\left\|\sigma_{k}^{\alpha} f\right\|_{p}^{p}}{k^{2-(1+\alpha) p}} \leq c_{\alpha, p}\|f\|_{H_{p}}^{p}
$$

Let $0<\alpha \leq 1, \beta>0$, and $\theta_{n}^{\alpha, \beta}$ denote the Nörlund mean, where

$$
\left\{q_{0}=0, q_{k}=k^{\alpha-1} \log ^{\beta} k: k \geq 1\right\} \text {, }
$$

that is,

$$
\theta_{n}^{\alpha, \beta} f=\frac{1}{Q_{n}} \sum_{k=1}^{n}(n-k)^{\alpha-1} S_{k} f .
$$

Remark $30<\alpha \leq 1$ and $\beta=0$. Then $\theta_{n}^{\alpha, \beta}$ satisfy conditions (3) and (4) and also conditions (11) and (12).

Remark $40<\alpha \leq 1$ and $\beta>0$. Then $\theta_{n}^{\alpha, \beta}$ satisfies conditions (11) and (12), but does not satisfy (3) and (4).

Finally, we also point out some new consequences of our results.

First we note that Theorem 1 and Remark 1 immediately imply the following new result.

\section{Corollary 6}

(a) Let $f \in H_{p}$, where $0<p<1 /(1+\alpha)$ for some $0<\alpha \leq 1$. Then for every $\beta>0$ the maximal operator

$$
\tilde{\theta}_{p}^{*, \alpha, \beta}:=\sup _{n \in \mathbb{N}} \frac{\left|\theta_{n}^{\alpha, \beta} f\right|}{(n+1)^{1 / p-1-\alpha}}
$$

is bounded from the martingale Hardy space $H_{p}$ to the Lebesgue space $L_{p}$.

(b) Let $\left\{\Phi_{n}: n \in \mathbb{N}_{+}\right\}$be any non-decreasing sequence, satisfying the condition

$$
\varlimsup_{n \rightarrow \infty} \frac{(n+1)^{1 / p-1-\alpha}}{\Phi_{n}}=\infty .
$$

Then

$$
\sup _{k \in \mathbb{N}} \frac{\theta_{M_{2 n_{k}+1} f_{k}}^{\alpha, \beta}\left\|_{M_{2 n_{k}+1}+1}\right\|_{\text {weak }-L_{p}}}{\left\|f_{k}\right\|_{H_{p}}}=\infty .
$$


In a similar way we see that Theorem 2 and Remark 1 immediately generates the following new result.

Corollary 7 Let $f \in H_{p}$, where $0<p<1 /(1+\alpha)$, for some $0<\alpha \leq 1$. Then for every $\beta>0$ there exists an absolute constant $c_{\alpha, \beta, p}$, depending only on $\alpha, \beta$, and $p$, such that

$$
\sum_{k=1}^{\infty} \frac{\left\|\theta_{k}^{\alpha, \beta} f\right\|_{p}^{p}}{k^{2-(1+\alpha) p}} \leq c_{\alpha, \beta, p}\|f\|_{H_{p}}^{p} .
$$

\section{Competing interests}

The authors declare that they have no competing interests.

\section{Authors' contributions}

All the authors contributed equally and significantly in writing this paper. All the authors read and approved the final manuscript.

\section{Author details}

'Department of Mathematics, Faculty of Exact and Natural Sciences, Ivane Javakhishvili Tbilisi State University, Chavchavadze str. 1, Tbilisi, 0128, Georgia. ${ }^{2}$ Department of Engineering Sciences and Mathematics, Luleå University of Technology, Luleå, 971 87, Sweden. ${ }^{3}$ UiT, The Artic University of Norway, P.O. Box 385, Narvik, 8505, Norway.

\section{Acknowledgements}

The research was supported by Shota Rustaveli National Science Foundation grants no. DO/24/5-100/14 and YS15-2.1.1-47, by a Swedish Institute scholarship no. 10374-2015 and by target scientific research programs grant for the students of faculty of Exact and Natural Sciences. The authors would like to thank the referees for helpful suggestions.

Received: 3 June 2016 Accepted: 20 September 2016 Published online: 01 October 2016

\section{References}

1. Schipp, F: Certain rearrangements of series in the Walsh system. Mat. Zametki 18(2), 193-201 (1975) (in Russian)

2. Pál, J, Simon, P: On a generalization of the concept of derivative. Acta Math. Acad. Sci. Hung. 29(1-2), 155-164 (1977)

3. Fujii, N: A maximal inequality for $H^{1}$-functions on a generalized Walsh-Paley group. Proc. Am. Math. Soc. 77(1), 111-116 (1979)

4. Simon, P: Investigations with respect to the Vilenkin system. Ann. Univ. Sci. Bp. Rolando Eötvös Nomin., Sect. Math. 27, 87-101 (1985)

5. Weisz, F: Cesàro summability of one- and two-dimensional Walsh-Fourier series. Anal. Math. 22(3), $229-242$ (1996)

6. Simon, P: Cesàro summability with respect to two-parameter Walsh systems. Monatshefte Math. 131(4), 321-334 (2000)

7. Goginava, U: The maximal operator of Marcinkiewicz-Fejér means of the $d$-dimensional Walsh-Fourier series. East J. Approx. 12(3), 295-302 (2006)

8. Tephnadze, G: Fejér means of Vilenkin-Fourier series. Studia Sci. Math. Hung. 49(1), 79-90 (2012)

9. Weisz, F: The Q-summability of Fourier series. Acta Math. Hung. 103(1-2), 139-175 (2004)

10. Weisz, F: $(C, \alpha)$ summability of Walsh-Fourier series. Anal. Math. 27(2), 141-156 (2001)

11. Goginava, U: The maximal operator of the $(C, \alpha)$ means of the Walsh-Fourier series. Ann. Univ. Sci. Bp. Rolando Eötvös Nomin., Sect. Comput. 26, 127-135 (2006)

12. Simon, P, Weisz, F: Weak inequalities for Cesàro and Riesz summability of Walsh-Fourier series. J. Approx. Theory 151(1), 1-19(2008)

13. Blahota, I, Tephnadze, G: On the $(C, \alpha)$-means with respect to the Walsh system. Anal. Math. 40, 161-174 (2014)

14. Blahota, I, Tephnadze, G, Toledo, R: Strong convergence theorem of Cesàro means with respect to the Walsh system. Tohoku Math. J. 67(4), 573-584 (2015)

15. Simon, P: Strong convergence theorem for Vilenkin-Fourier series. J. Math. Anal. Appl. 245, 52-68 (2000)

16. Gát, G: Investigations of certain operators with respect to the Vilenkin system. Acta Math. Hung. 61, 131-149 (1993)

17. Tephnadze, G: On the partial sums of Vilenkin-Fourier series. J. Contemp. Math. Anal. 49(1), 23-32 (2014)

18. Tephnadze, G: Strong convergence theorems of Walsh-Fejér means. Acta Math. Hung. 142(1)، 244-259 (2014)

19. Tephnadze, G: A note of the Fourier coefficients and partial sums of Vilenkin-Fourier series. Acta Math. Acad. Paedagog. Nyházi. 28, 167-176 (2012)

20. Blahota, I, Tephnadze, G: Strong convergence theorem for Vilenkin-Fejér means. Publ. Math. (Debr.) 85(1-2), 181-196 (2014)

21. Móricz, F, Siddiqi, A: Approximation by Nörlund means of Walsh-Fourier series. J. Approx. Theory 70(3), 375-389 (1992)

22. Blahota, I, Gát, G: Norm summability of Nörlund logarithmic means on unbounded Vilenkin groups. Anal. Theory Appl. 24(1), 1-17 (2008)

23. Nagy, K: Approximation by Nörlund means of quadratical partial sums of double Walsh-Fourier series. Anal. Math 36(4), 299-319 (2010)

24. Nagy, K: Approximation by Nörlund means of double Walsh-Fourier series for Lipschitz functions. Math. Inequal. Appl. 15(2), 301-322 (2012)

25. Nagy, K: Approximation by Nörlund means of Walsh-Kaczmarz-Fourier series. Georgian Math. J. 18(1), 147-162 (2011) 
26. Nagy, K: Approximation by Cesàro means of negative order of Walsh-Kaczmarz-Fourier series. East J. Approx. 16(3), 297-311 (2010)

27. Blahota, I, Persson, LE, Tephnadze, G: On the Nörlund means of Vilenkin-Fourier series. Czechoslov. Math. J. 65(4), 983-1002 (2015)

28. Blahota, I, Tephnadze, G: A note on maximal operators of Vilenkin-Nörlund means. Acta Math. Acad. Paedagog. Nyházi. 32, 203-213 (2016)

29. Memić, N, Persson, LE, Tephnadze, G: A note on the maximal operators of Vilenkin-Nörlund means with non-increasing coefficients. Studia Sci. Math. Hung. doi:10.1556/012.2016.1342

30. Vilenkin, NY: On a class of complete orthonormal systems. Transl. Am. Math. Soc. 28(2), 1-35 (1963)

31. Weisz, F: Martingale Hardy Spaces and Their Applications in Fourier Analysis. Lecture Notes in Mathematics, vol. 1568 Springer, Berlin (1994)

32. Moore, CN: Summable Series and Convergence Factors. Dover, New York (1966)

33. Gát, G: Cesàro means of integrable functions with respect to unbounded Vilenkin systems. J. Approx. Theory 124(1), 25-43 (2003)

34. Weisz, F: Hardy spaces and Cesàro means of two-dimensional Fourier series. Bolyai Soc. Math. Stud. 5, 353-367 (1996)

35. Tephnadze, G: On the maximal operators of Vilenkin-Fejér means on Hardy spaces. Math. Inequal. Appl. 16(2), 301-312(2013)

\section{Submit your manuscript to a SpringerOpen ${ }^{\ominus}$ journal and benefit from:}

- Convenient online submission

- Rigorous peer review

- Immediate publication on acceptance

- Open access: articles freely available online

- High visibility within the field

- Retaining the copyright to your article 